\title{
ISONEBULA ACANTHOPLEON SP.N. (ISOPODA, CYMOTHOIDAE) NUEVO ECTOPARÁSITO DE PECES CURIMÁTIDOS
}

\author{
Roberto Taberner ${ }^{1}$
}

\begin{abstract}
ISONEBUL.4 ACANTHOPLEON SP.N. (ISOPODA, CYMOTHOIDAE) A NEW ECTOPARASIT OF CURIMATIDAE. Isonebula acanthopleon a new species is described and illustrated from specimens of both sexes. The species name refers to the pointed process of the $5^{\text {th }}$ pleonite. Its known hosts are Cyphocharax platanus (Günther, 1880), C. voga (Hensel, 1869) and C. spilotus (Vari, 1987) of the family Curitmatidae (Pisces).

KEY WORDS. Isopoda, Cymothoidae, Isonebula acantopleon, new species, ectoparasit, Curimatidae
\end{abstract}

TABERNER (1977) instituyó Nebula maculatus gen.n. y sp.n. (Isopoda, Cymothoidae) ectoparásito del pez Metynis maculatus (Kner, 1860) Berg, 1897. Notificado de que el nombre genérico era preocupado por Lepidoptera, en TABERNER (1979a) publicó la aclaración y propuso el nuevo nombre de Isonebula y como es femenino, la composición resultó ser I. maculata. dicho género se caracteriza y diferencia de los otros sudamericanos de agua dulce, por tener el céfalon con la frente redondeada y los bordes del pleotelson subparalelos. En este trabajo, se describe una nueva especie a la que se ha denominado Isonebula acanthopleon por cuanto posee un proceso en punta en el borde posterior del pleonito 5 .

Un sólo ejemplar era conocido desde hace mucho tiempo encontrado en Cyphocharax platanus (Günther, 1880), sin embargo, no se publicó su descripción a la espera de nuevos ejemplares para poder realizar el estudio con mayor precisión y clasificarla con exactitud.

La oportuna recepción de muestras de curimátidos, conjuntamente con otra cantidad de especies ícticas, algunos de ellos parasitados, enviados por la Prof. Ely Cordiviola de Yuan (Instituto Nacional de Limnología, Santo Tomé, provincia de Santa Fe, Argentina); ha permitido completar éste trabajo.

\section{Isonebula acanthopleon sp.n.}

Figs 1-38

Holotipo. MACN no 33997 (Fig. 22-23), 1 ejemplar en fase hembra, de cuerpo ovoide y algo alargado en vista dorsal, de $11,8 \mathrm{~mm}$ de longitud total y $6,8 \mathrm{~mm}$ de ancho máximo a nivel del pereonito 4 . Obtenido en el Rio de la Plata, Capital

1) Museo Argentino de Ciencias Naturales Bernardino Rivadavia. Avenida Angel Gallardo 470 , 1405 Buenos Aires, Argentina. 


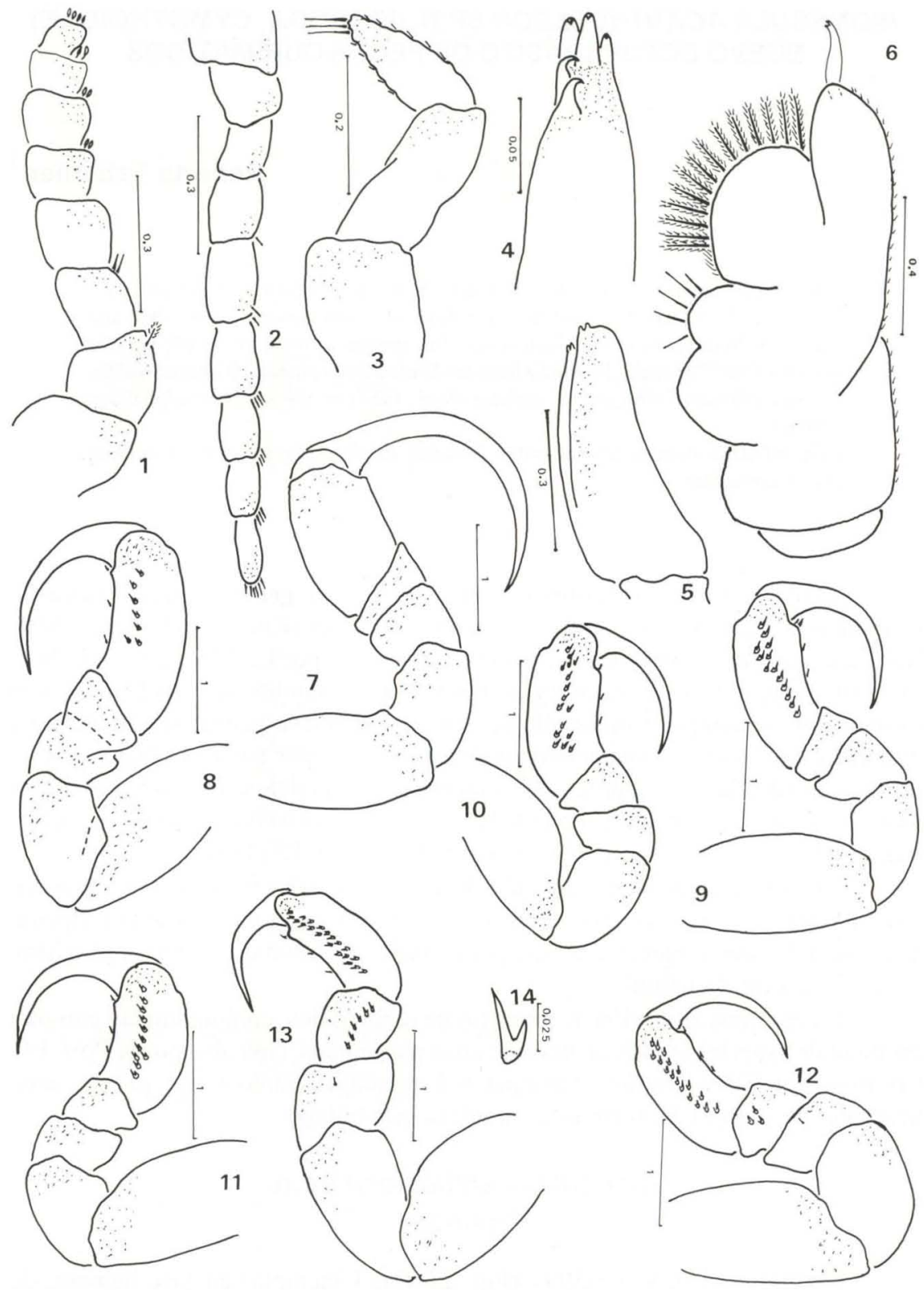

Figs 1-14. Isonebula acanthopleon sp.n., individuo en fase hembra. (1) Anténula; (2) antena; (3) palpo mandibular; (4) maxilula; (5) maxila; (6) maxilipedio; (7-13) pereópodos 1 a 7 , respectivamente; (14) espinas dobles. 


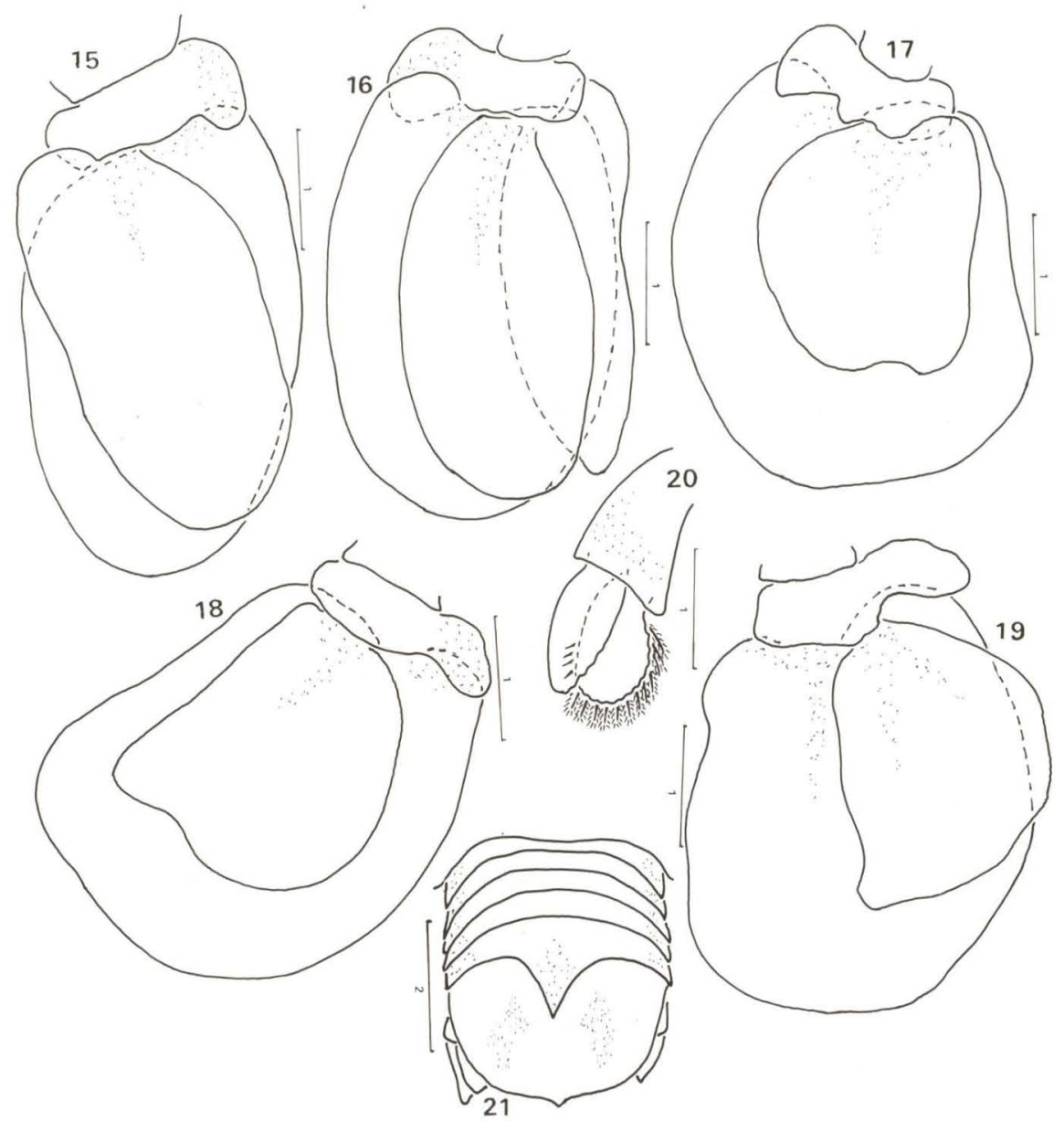

Figs 15-21. Isonebula acanthopleon sp.n., individuo en fase hembra. (15-19) Pleópodo 1 a 5 , respectivamente; (20) urópodo; (21) vista dorsal del pleon.

Federal, sin fecha de captura; R. Castillo leg.. Parasitando un ejemplar de Cyphocharax platanus (Günther, 1880) de la colección del MACN n 4008 de la Sección Ictiología. Fijado en formol al 10\% y conservado en alcohol al $70 \%$.

Céfalon. De 1,8mm de largo, triangular, algo inmerso en el pereonito 1, de frente ancha, redondeada y algo inclinada hacia abajo, 1,2 veces tan ancho como largo. Ojos en la parte posterior de los bordes laterales, medianos y subredondeados. Anténulas de 8 artejos, más cortas y gruesas que las antenas que tienen nuove artejos.

Pereon. Su longitud contenida 2,1 veces en el largo total. Pereonitos algo convexos y comprimidos hacia adelante, convexos del 4 al 7. El pereonito 1 es el más largo con el margen anterior algo trisinuado, le sigue el 3 y los demás subiguales. Bordes laterales redondeados, ángulos posteriores del 3 al 7 agudos. Los epímeros se observan en vista dorsal ocupando todo el borde lateral de los pereonitos 6 y 7 . 

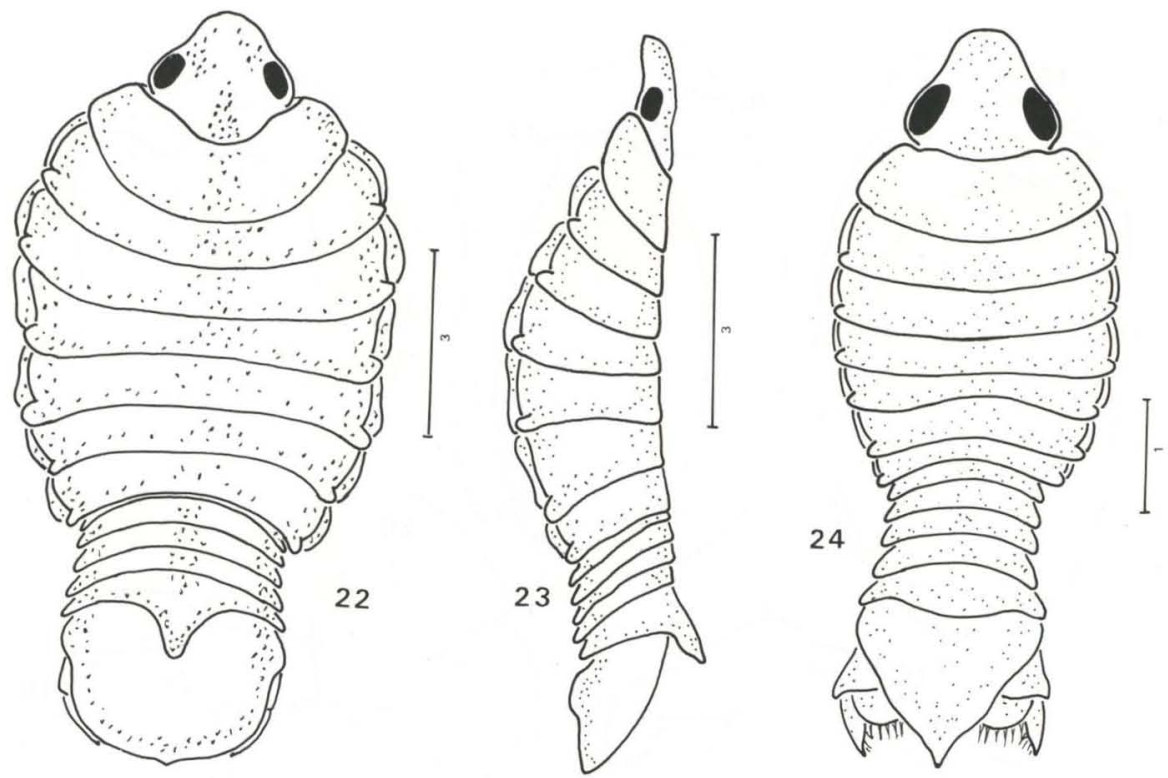

Figs 22-24. Isonebula acanthopleon sp.n.. (22) Individuo en fase hembra, vista dorsal; (23) vista lateral; (24) individuo en fase macho, vista dorsal.

Pleotelson. De bordes subparalelos, su longitud contenida 2,7 veces en el largo total. El pleonito 1 algo inmerso en el pereonito 7, pleonito 2 a 4 subiguales, pleonito 5 es el más largo con el borde posterior extendido hacia atrás y separado del telson formando una punta aguda (Figs 21, 23). Telson subredondeado, borde posterior en forma de quilla.

Anténulas (Fig. 1). Cilíndricas, de 8 artejos, extendidas alcanzan casi el borde posterior del ojo. El artejo 1 es el más grande en tamaño, le siguen el 2, 3 y 4; luego el 5, 6 y 7; el 8 es el más pequeño. El artejo 2 con una y el 3 con dos sedas; el 5, 6, 7 y 8 con estetascos todos ellos en la zona distal.

Antenas (Fig. 2). Cilíndricas de nuove artejos, extendidas alcanzan la mitad del borde lateral del pereonito 1 . El artejo 1 es el más grande, los demás son subiguales en longitud pero se van angostando hacia el extremo. Los artejos 3 y 4 con una seda apical, el 5 con dos, el 6, 7 y 8 con tres y el 9 con seis.

Mandibula (Fig. 3). Palpo mandibular con tres segmentos, el distal con pequeños pelos en su superficie y tres terminales más largos.

Maxílula (Fig. 4). Con tres dientes terminales y dos por debajo.

Maxila (Fig. 5). Formada por dos láminas con dos dientes pequeños distales en cada una.

Maxilipedio (Fig. 6). Lamela interna trilobulada, el lóbulo distal es el de mayor tamaño con 14 sedas plumosas en el borde, el intermedio con cuatro pelos. Palpos de dos artejos, el proximal subovalado con dos dientes en el extremo.

Pereópodo 1 (Fig. 7). Es el de mayor tamaño, la uña muy larga y principal elemento de fijación al hospedados, llega al borde distal del ischio. 

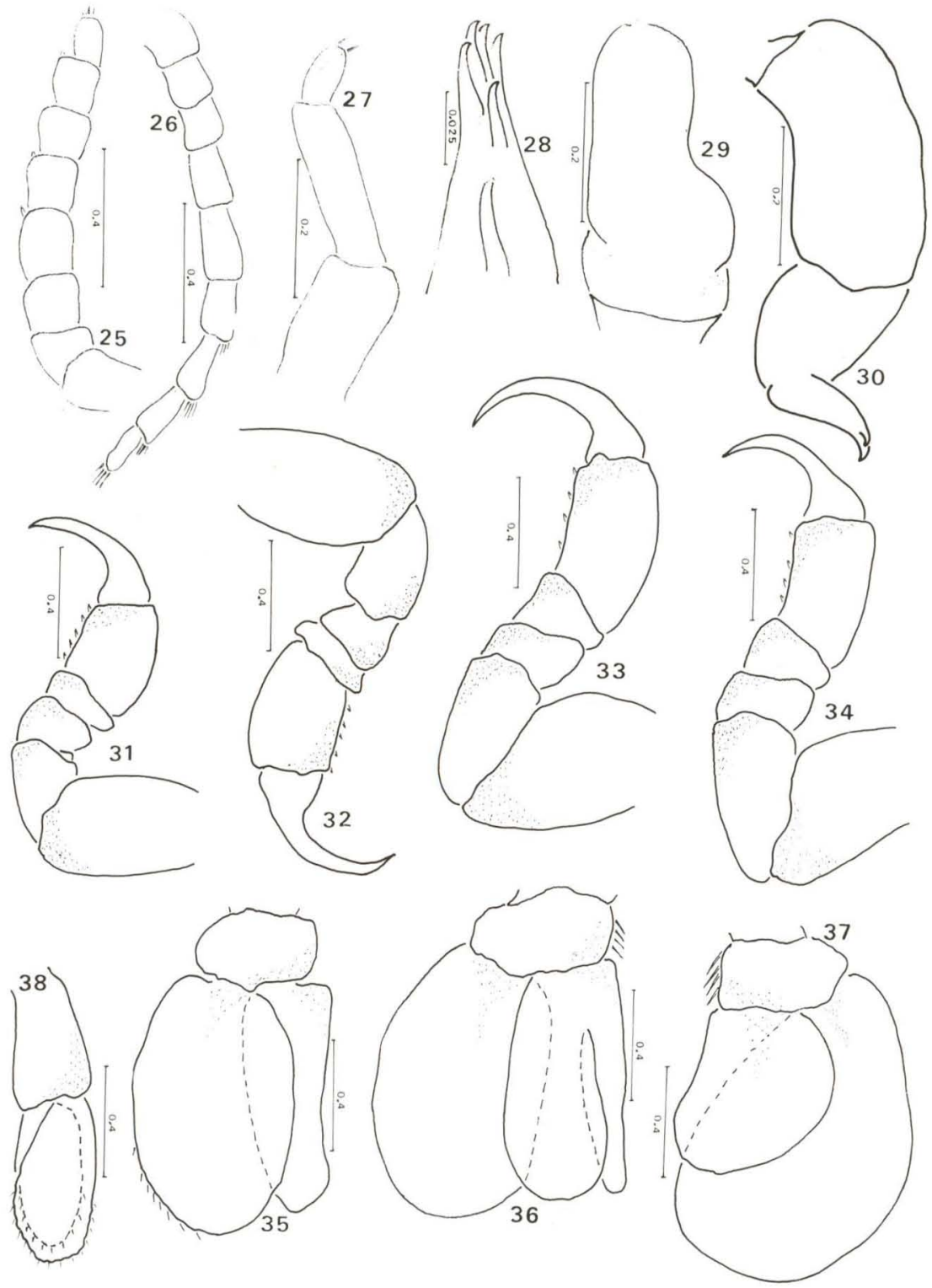

Figs 25-38. Isonebula acanthopleon sp.n., individuo en fase macho. (25) Anténula; (26) antena; (27) palpo mandibular; (28) maxílula; (29) maxila; (30) maxilipedio; (31) pereópodo 1; (32) pereópodo 2 ; (33) pereópodo 5 ; (34) pereópodo 7; (35) pleópodo 1 ; (36) pleópodo 2 ; (37) pleópodo 3; (38) urópodo.

Pereópodos 2 a 7 (Figs 8-13). Son subiguales, algo menores en tamaño que el pereópodo 1. Uñas medianas excepto en el pereópodo 7 que son pequeñas. El própodo de los pares 2 a 7 con una fila de espinas dobles (Fig. 14), como asi también el carpo del 6 y $7, y$ una simple en el mero. 
Pleópodos 1 a 5 (Figs 15-19). Basipodito subcuadrangular con un lóbulo pequeño en el margen externo ambas ramas laminares, la interna más pequeña el pleópodo 2 presenta los restos del estilete copulador.

Urópodos (Fig. 20). El basipodito algo más largo que ancho. Exopodito subovoide sin sedas, el endopodito un poco más ancho que el exopodito, con sedas plumosas en número de 16 aproximadamente.

Color. Blanco pardusco.

Material examinado. El holotipo y los seguientes ejemplares: Alotipo - Santa $\mathrm{Fe}$ : laguna de la isla "El Espinillar", cerca del rio Colastiné, 1 macho de 7,0mm de longitud total y $2,8 \mathrm{~mm}$ de ancho máximo, parasitando a Cyphocharax spilotus (Vari, 1987), 31-VIII-1991, E. Cordiviola leg., MACN no 33998 (Figs 24-38). Paratipos -La Plata: Rio de la Plata, 1 hembra de 9,0mm de longitud total y 4,0mm de ancho máximo, parasitando a Cyphocharax planatus, 26-X-1948, Z. Popovici leg., MACN $n^{0}$ 33999. Santa Fe: laguna en la isla "Los Sapos", 21-XI-1991, E. Cordiviola leg.: 1 hembra de $12,8 \mathrm{~mm}$ de longitud total y $7,0 \mathrm{~mm}$ de ancho máximo, parasitando a Cyphocharax voga (Hensel, 1869), MACN n $34000 ; 3$ hembras entre 7,0 y 9,5mm de longitd total y entre 3,8 y $5,4 \mathrm{~mm}$ de ancho máximo; 1 macho de $5,1 \mathrm{~mm}$ de longitud total y $3,0 \mathrm{~mm}$ de ancho máximo, parasitando a C. spilotus, MACN n ${ }^{\circ}$ 34001. Santa Fe: laguna en la isla "El Espinillar", cerca del rio Colastiné, 9-I-1992, E. Cordiviola leg.: 1 hembra de 8,5mm de largo total y 4,0 $\mathrm{mm}$ de ancho máximo, parasitando C. platanus, MACN n ${ }^{\circ} 34002 ; 2$ hembras entre 8,8 y $9,0 \mathrm{~mm}$ de longitud total y 4,0mm de ancho máximo, parasitando C. spilotus, MACN nº 34003.

\section{DISCUSIÓN}

La nueva especie la separan de I. maculata por algunas diferencias: en $I$. acanthopleon el cuerpo es deprimido en los primeros pereonitos y convexo hacia atrás, más evidente en los 4 últimos. La frente proporcionalmente más ancha y redondeada. El pleon presenta en el borde posterior del pleonito 5 (Figs 21, 23), una extención separada del telson en forma aguda.

Como se menciona más arriba, hacía tiempo que se conocía éste especímen, sin embargo, con la afluencia de muestras de peces ahora estudiadas, se logró obtener mayor cantidad de parásitos que permitió localizar los hospedadores y su ubicación. Para tal fin, fue necesario la revisión de diversas especies de characínidos y cíchlidos para concluir que solamente se lo encontró en curimátidos y dentro de éste grupo sólo en tres especies que se incluyen dentro del género Cyphocharax Fowler, 1906. Las especies son C. platanus (Günther, 1880); C. spilotus (Vari, 1987) y C. voga (Hensel, 1869). Para la determinación de los taxones ícticos se ha consultado el trabajo de VARI (1992).

Isonebula acanthopleon, en su faz hembra adulta, se ubica en la boca con el céphalon hacia atrás igual que su congénere $I$. maculata como fue indicado en TABERNER (1979b). Se prende con el primer par de uñas, más desarrolladas y se ayuda con las demás. Otro elemento que utiliza como fijación es el estilete que sale de la parte posterior del pleonito 5, éste lo traba en el paladar del hospedador y hace difícil su desprendimento. 
AGRADECIMIENTOS. El autor agradece a la Prof. Ely Cordiviola de Yuan y a su equipo, por la paciente constancia en los envios, como así también, a las autoridades del Museo Argentino de Ciencias Naturales "Bernardino Rivadavia" por permitir la consulta de la colección y al Lic. Ricardo Palma del Museum of New Zealand, por sus observaciones y la lectura critica del manuscrito.

\section{REFERENCIAS BIBLIOGRÁFICAS}

BERG, C. 1897. Contribución al conocimiento de los peces sudamericanos especialmente de los de la República Argentina. An. Mus. Nac. Buenos Aires 5: 263-302.

FOWLER, H.W. 1906. Further knowledge of some heterognathus fishes. Part I. Proc. Acad. Nat. Scie. Philad. 58: 293-351.

GÜNTHER, A. 1880. A contribution to the knowledge of the fish-fauna of the Rio de la Plata. Ann. Mag. Nat. Hist. 6 (5): 7-15.

Hensel, R.H. 1869. The freshwater fishes of Southern Brazil. Arch. Nat., Wiegmann, 36 (1): 50-91.

KNER, R. 1860. Zur familie der Characinen. III. Folge der Ichthyologischen Beiträge. Sitz. Akad. Wiss. Wien. 18: 9-62.

TABERNER, R. 1977. Nebula maculatus gen. nov. sp. nov. (Crustacea, Isopoda, Cymothoidae) ectoparásito de Metynis maculatus (Kner, 1860) Berg, 1897 (Pisces, Characidae). Physis 36 (92B): 141-146.

1979a. Aclaración sobre el nombre Nebula maculatus Taberner, 1977 (Crustacea, Isopoda, Cymothoidae). Physis 38 (94B): 55.

1979b. Datos para el conocimiento biológico de Paracymothoa parva Taberner, 1976 e Isonebula maculata Taberner, 1977 (Crustacea, Isopoda, Cymothoidae). Rev. Inst. Nac. Inv. C. Nat., Parasitología, 2 (4): 77-86.

VARI, R. 1987. Two new species of curimatid fiches (Ostariophysi: Characiformes) from Rio Grande do Sul, Brazil. Proc. Biol. Soc. Wash. 100 (3): 603-609.

1992. Systematics of the neotropical characiform genus Cyphocharax Fowler (Pisces: Ostariophysi). Smith. Contr. Zool. 529: 1-137. 